$>$ Les données issues du décryptage du génome humain puis de la protéomique naissante doivent être intégrées dans les structures de la cellule vivante si l'on veut parvenir à une meilleure compréhension de son fonctionnement normal et pathologique. L'organisation compartimentée de la cellule eucaryote, pressentie dès la fin du $\mathrm{XIX}$ siècle, est maintenant définitivement établie dans l'ensemble du monde vivant. Cet article situe dans une perspective historique les données de base indispensables à la compréhension des recherches modernes sur les mécanismes du transport intracellulaire. <

\section{Les compartiments membranaires de la cellule eucaryote}

Andrée Tixier-Vidal

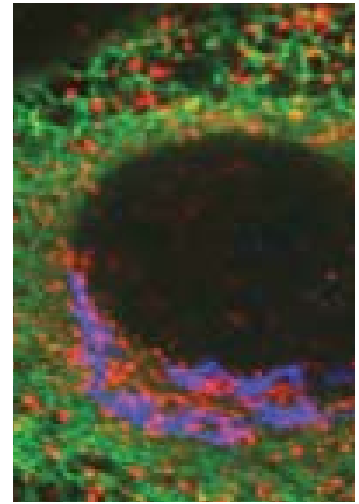

NBSI, Cnrs UMR 7624, Université

Pierre et Marie Curie, 7, quai Saint-Bernard, 75252 Paris Cedex 05, France.
L'existence d'un réseau membranaire au sein des cellules eucaryotes a été révélée à partir des années 1950 avec l'avènement du microscope électronique. Auparavant, l'examen au microscope optique de coupes pratiquées dans des tissus présentant une différenciation morphologique propice (cellules glandulaires, tissus nerveux, ...) avait conduit des observateurs particulièrement perspicaces et imaginatifs à identifier des structures intracellulaires spécialisées telles que l'ergastoplasme décrit par Garnier en 1899 [1] dans des glandes salivaires humaines, et l'appareil de Golgi décrit en 1898 par Golgi dans des cellules nerveuses ganglionnaires [2] (Figure 1). Cependant, le rôle de ces structures restait purement spéculatif et leur existence, tributaire de l'emploi de techniques tinctoriales empiriques, a été controversée pendant toute la première moitié du XXe siècle. Dès 1948, l'examen de fibroblastes en culture à l'aide des premiers microscopes électroniques suggéra l'existence d'un réseau tubulaire membranaire intracellulaire, le réticulum endoplasmique, dont l'existence fut ensuite confirmée par l'examen de coupes ultrafines. Ce réseau tubulaire présentait une différenciation morphologique qui fut identifiée par Dalton [3] comme étant l'appareil décrit antérieurement par Golgi. Au cours de la décennie suivante, les progrès des techniques de microscopie électronique et l'examen de types cellulaires très variés ont confirmé la réalité du réticulum endoplasmique et sa subdivision en compartiments morphologiquement distincts. Cela a préparé l'analyse fonctionnelle et biochimique de ces structures qui débuta à partir de 1963 avec les travaux de Leblond, au Canada, et ceux de Palade aux ÉtatsUnis. Ce dernier, qui obtint, avec Claude et De Duve, le Prix Nobel de Physiologie et Médecine en 1974, établit le paradigme du transport intracellulaire des protéines [4]. Prenant comme modèle la cellule exocrine du pancréas, il postula que la protéine néo-synthétisée dans le cytoplasme au niveau des ribosomes est transportée dans la lumière du réticulum endoplasmique puis parcourt successivement plusieurs compartiments membranaires. Elle pénètre d'abord dans le réticulum endoplasmique rugueux, ainsi nommé parce qu'il est tapissé sur sa face cytoplasmique de ribosomes (ce compartiment fut identifié par Hagueneau [5] comme étant l'ergastoplasme décrit par Garnier). La protéine atteint ensuite un ensemble de structures membranaires lisses, I'appareil de Golgi. À sa sortie, elle est concentrée dans des granules entourés d'une membrane lisse. Ceux-ci sont stockés temporairement dans le cytoplasme avant de libérer leur contenu à l'extérieur de la cellule par un 
processus complexe décrit sous le terme d'exocytose. La membrane du granule entre en contact avec la face cytoplasmique de la membrane plasmique. La fusion ponctuelle des deux membranes permet la libération du contenu des granules dans le milieu extracellulaire.La membrane du granule est alors temporairement intégrée à la membrane plasmique. Dans les cellules non glandulaires, le même mécanisme d'exocytose a été retrouvé pour des petites vésicules claires qui jouent un rôle majeur dans la construction et le renouvellement de la membrane plasmique.

Ce concept du transport intracellulaire des protéines reposait sur des bases expérimentales solides apportées par l'autoradiographie à haute résolution qui permet de localiser, en microscopie électronique, les protéines ayant incorporé un acide aminé radioactif et de suivre leur trajet intracellulaire en fonction du temps écoulé depuis leur synthèse. En revanche, les mécanismes mis en jeu au cours de ce transport étaient purement hypothétiques. Deux questions fondamentales étaient posées : quel est le devenir de la protéine au cours de son voyage intra-luminal ? Et quels sont les mécanismes assurant la communication entre les différents compartiments? Dès les années 1970, Palade [4] a émis l'hypothèse selon laquelle ceux-ci communiqueraient de façon intermittente par l'intermédiaire d'une «navette »de vésicules. Ce phénomène impliquerait trois étapes : la formation d'un bourgeon à partir d'un compartiment «donneur », la fission du bourgeon en une vésicule et la fusion de la vésicule avec un compartiment « accepteur » selon un processus analogue à celui de l'exocytose.

L’hypothèse vésiculaire a rapidement été étendue à tous les mouvements membranaires intracellulaires, quelle que soit leur direction, centrifuge ou centripète. Les premiers constituent la « voie de biosynthèse-exocytose » empruntée par les protéines solubles destinées à être exportées hors de la cellule ainsi que par les protéines membranaires qui seront incorporées dans la membrane plasmique. Les seconds constituent la «voie d'endocytose » assurant l'entrée dans la cellule de macromolécules provenant du milieu extracellulaire. L'hypothèse vésiculaire est à la base de toutes les recherches ultérieures visant à élucider les mécanismes moléculaires du transport intracellulaire. Elle est à l'origine de la découverte des nombreux gènes impliqués dans le transport intracellulaire dans toutes les cellules eucaryotes. Cependant, dès sa formulation, elle est entrée en conflit avec des hypothèses alternatives, postulant la communication continue par des tubules et la progression-maturation des compartiments. À l'heure actuelle, les recherches s'appuyant sur de nouvelles techniques d'imagerie cellulaire applicables à des cellules vivantes [6] privilégient un transport tubulaire compatible avec les données moléculaires déjà acquises. De plus, des expériences récentes ont apporté de nouveaux arguments en faveur du mécanisme de progression-maturation, au moins dans certains cas $[7,8]$. Au cours du dernier quart du XXe siècle, des progrès considérables ont été accomplis dans la compréhension de ces mécanismes. À l'heure actuelle, les découvertes s'accumulent de façon exponentielle, levant de nouvelles interrogations, suscitant la mise en œuvre de techniques de plus en plus performantes, faisant appel à tous les domaines des sciences du vivant. Cet article a pour objectif d'établir les bases structurales nécessaires à la compréhension des recherches récentes.

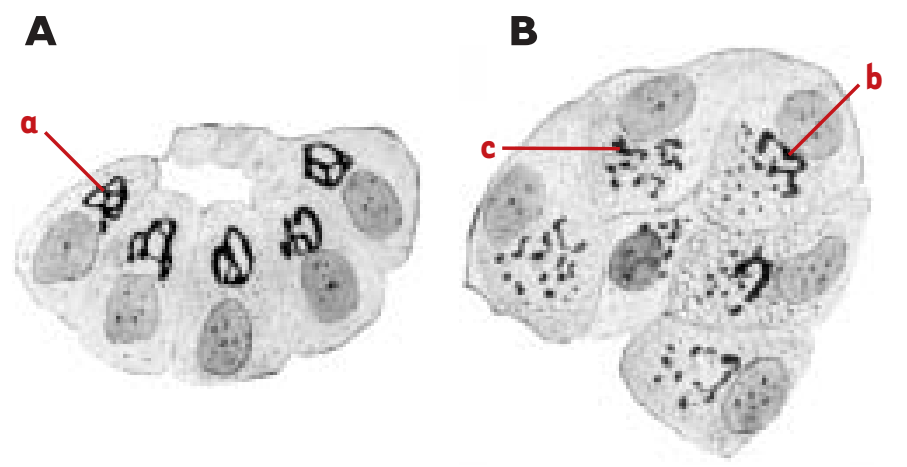

Figure 1. Appareil de Golgi observé en microscopie optique dans les cellules du pancréas exocrine de jeune lapin. Dans ce dessin original, Cajal met en évidence les variations de la forme de l'appareil de Golgi qui apparaît en noir par la technique de Golgi $(a, b, c)$ dans une cellule pauvre $(A)$ ou riche $(B)$ en grains de zymogène (d'après [29]).

\section{Les compartiments membranaires sont définis par leur structure et leur fonction}

Succédant à la définition purement morphologique des compartiments membranaires, les recherches ont eu pour objectif de déterminer leurs compositions biochimiques et leurs fonctions respectives. Deux approches techniques complémentaires ont contribué à l'amélioration des connaissances : le fractionnement subcellulaire et l'identification cytochimique dans des cellules intactes par autoradiographie et immunocytochimie ultrastructurales. Ces données se sont ensuite enrichies de l'apport de la génétique cellulaire et de la biologie moléculaire qui ont révélé l'unicité d'organisation des cellules eucaryotes dans l'ensemble du monde vivant. Progressivement, une vision dynamique des compartiments membranaires s'est substituée à la conception statique initiale. En conséquence, la définition, en 
termes moléculaires, de ces compartiments pose encore des problèmes [9]. En effet, les compartiments cellulaires étant en communication, leurs frontières sont parfois mal définies et varient en fonction de l'activité et de la spécialisation fonctionnelle des cellules. Néanmoins, on considère que chaque compartiment membranaire possède une majorité de composants qui lui sont spécifiques.

\section{Les membranes biologiques sont une mosaïque de protéines et de lipides}

Les membranes biologiques sont composées de lipides (30 - $50 \%$ en moyenne) et de protéines (70 - $50 \%$ ) [10, 11].

Les lipides membranaires constituent la structure de base des membranes. Du fait de leur nature amphiphile, ils s'organisent spontanément en une double couche : les têtes polaires sont en contact avec le milieu aqueux (extracellulaire ou intracellulaire) et les chaînes hydrophobes s'associent face à face. Les lipides membranaires comprennent en majorité des phospholipides, le plus abondant étant la phosphatidylcholine ; il s'y ajoute du cholestérol et des glycosphingolipides. Leur nature et leurs proportions respectives varient à la fois le long des compartiments membranaires et en fonction de la différenciation cellulaire. On sait maintenant qu'ils sont organisés en microdomaines de composition et de rigidité distinctes. Ces microdomaines sont mobiles dans le plan de la membrane où ils forment des « radeaux lipidiques » enrichis en cholestérol et en sphingolipides [12]. La diversité de la distribution des phospholipides membranaires est assurée par des mécanismes spécifiques qui commencent seulement à être compris [13]. Le rôle des lipides membranaires dans le fonctionnement cellulaire est double. Outre leur participation dans le transport membranaire et le tri des protéines, ils sont impliqués dans la transduction des signaux extracellulaires activant des récepteurs spécifiques de la membrane plasmique $[12,13]$. De ce fait, ils interviennent vraisemblablement dans le couplage de ces deux types d'événements.

Les protéines membranaires sont soit intégrées dans la bicouche lipidique (protéines membranaires intégrales ou transmembranaires), soit associées à l'une des faces de la bicouche lipidique (protéines dites périphériques). Cette distinction repose sur des mécanismes de biosynthèse et d'insertion différents $[10,11]$. Les protéines dites périphériques sont associées à l'une des faces de la bicouche lipidique par des mécanismes enzymatiques assurant l'addition covalente de chaînes lipidiques. Les protéines associées à la face cytoplasmique du réticulum sont synthétisées dans le cytosol et peuvent subir différentes modifications selon la nature des chaînes lipidiques ajoutées (myristoylation, palmitoylation, isoprénylation). D’autres protéines sont associées dans la face luminale du réticulum et, après insertion dans la membrane plasmique, elles sont exposées à la surface cellulaire. Parmi celles-ci on citera les protéines ancrées par un groupement glycosyl-phosphatidylinositol (GPI) [10, 11]. De même que les lipides, les protéines membranaires sont mobiles latéralement, conformément au modèle de mosaïque fluide proposé dès 1972 par Singer et Nicholson [14]. Cependant, ces mouvements ne se font pas au hasard. Par exemple, les protéines périphériques pourvues d'un groupement GPI

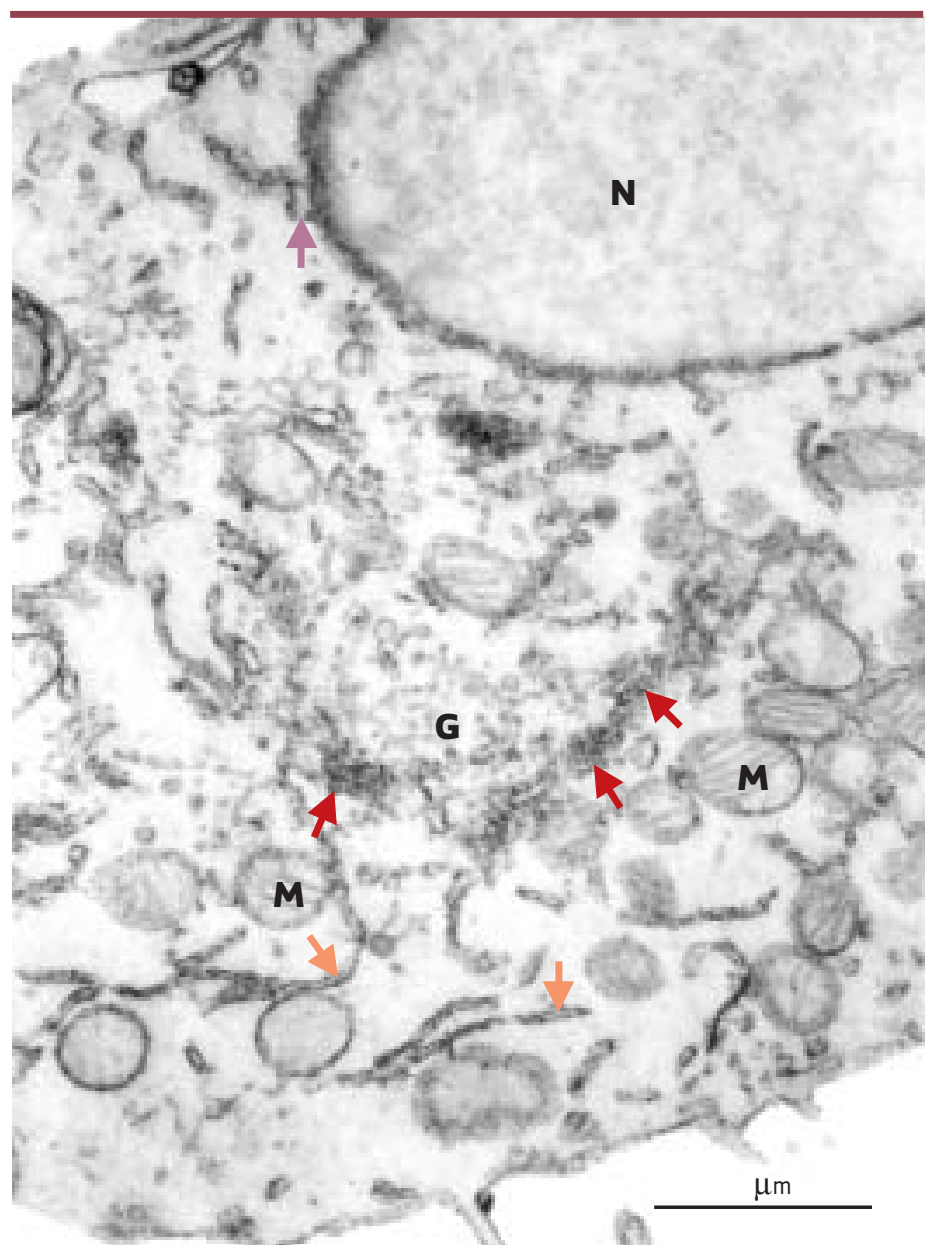

Figure 2. Aspect en microscopie électronique de l'ensemble du réticulum membranaire. Le réticulum est révélé par une technique d'imprégnation des membranes dans une coupe fine de cellules GH3, lignée de cellules antéhypophysaires sécrétant de la prolactine. 0 n observe les nombreuses citernes linéaires du réticulum rugueux (flèches oranges) et leurs communications avec la citerne périnucléaire (flèche violette). L'appareil de Golgi (G) est reconnu par sa forme semi-circulaire et par l'empilement de fines citernes parallèles (flèches rouges) entourant une zone riche en vésicules. On remarque la complexité de l'organisation tubulo-vésiculaire sur les faces cis et trans. M : mitochondries; $N$ : noyau. (cliché original A. Tixier-Vidal). 
ont une affinité pour les radeaux lipidiques [12]. Des mécanismes contrôlent ces mouvements latéraux qui jouent un rôle déterminant dans le trafic membranaire, le transport et l'adressage des protéines [13] de même que dans le couplage des mouvements membranaires avec le cytosquelette et la signalisation intracellulaire.

\section{Le réticulum endoplasmique rugueux assure la synthèse et contrôle la qualité des protéines exportées}

Le réticulum endoplasmique rugueux est généralement désigné, à l'exemple des auteurs anglo-saxons, sous le terme de réticulum endoplasmique. Nous adoptons ici cette nomenclature bien qu'elle ne recouvre pas la réalité morphologique que nous avons citée au début de cet article. En amont, le réticulum endoplasmique est en continuité avec la citerne périnucléaire. En aval, il communique avec le compartiment suivant, l'appareil de Golgi, par un

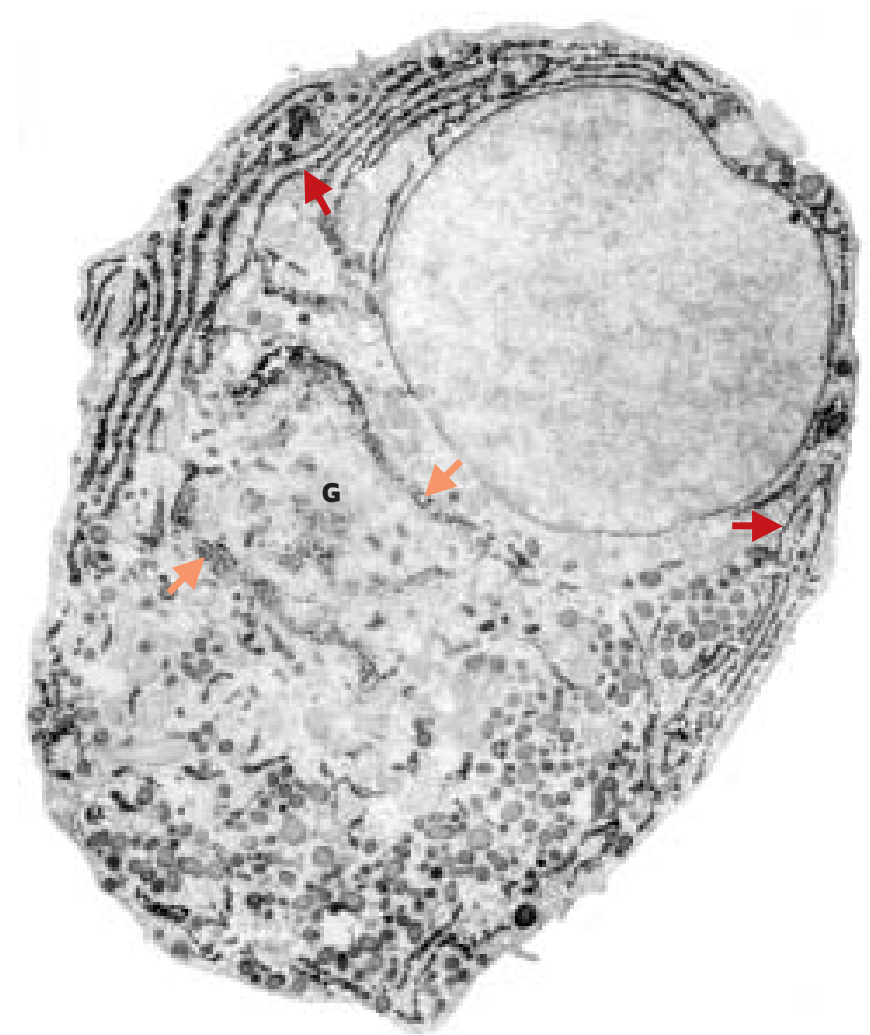

Figure 3. Détection de la prolactine dans une cellule glandulaire de l'anté-hypophyse de rat observée en microscopie électronique. L'ensemble du réticulum est révélé par la localisation immunocyto-enzymologique de son contenu, la prolactine. Les citernes du réticulum endoplasmique (flèches rouges) communiquent avec la citerne périnucléaire. Flèches oranges : saccules aplatis et curvilignes de l'appareil de Golgi (G)(d'après [30]). ensemble de structures tubulo-vésiculaires plus ou moins développées (Figures 2-4).

Le réticulum endoplasmique assure plusieurs fonctions indispensables à la vie de la cellule qui sont résumées très brièvement. II est le siège de la synthèse de tous les composants membranaires, lipides, phospholipides et protéines. Les lipides et les phospholipides sont synthétisés par des enzymes membranaires à partir des éléments solubles présents dans le cytosol alors que les protéines membranaires sont synthétisées à partir d'acides aminés libres du cytosol par la traduction d'ARN messagers au niveau des ribosomes. Les protéines $\rightarrow) \mathrm{m} / \mathrm{s}$

$1999, n^{\circ} 11$, p. 1336 nismes complexes $[10,11]$.

Le réticulum endoplasmique assure la synthèse et le contrôle de la qualité des protéines destinées à être exportées. Les chaînes polypeptidiques, dont la synthèse ribosomique débute dans le cytosol par une séquence d'acides aminés hydrophobes (séquence signal), sont dirigées vers la membrane du réticulum endoplasmique. Cela entraîne la liaison des ribosomes à la face cytoplasmique du réticulum endoplasmique et le transport de la chaîne naissante dans la lumière du réticulum endoplasmique où la coupure de la séquence signal par une peptidase libère la protéine dans la lumière du réticulum endoplasmique. Les mécanismes moléculaires de ce ciblage des protéines transportées ont été disséqués de manière approfondie par Blobel (Prix Nobel de Médecine, 1999) $(\rightarrow)$ et ses élèves [15]. Le contrôle de la qualité des protéines exportées est assuré par un ensemble de réactions se déroulant dans la lumière du réticulum, soit pendant l'allongement de la chaîne (modifications cotraductionnelles) soit après (modifications post-traductionnelles). Ces modifications sont assurées par des enzymes membranaires ou luminales. Elles consistent principalement en trois types de réactions : des clivages peptidiques, le démarrage de la glycosylation par ajout à un résidu asparagine d'un oligosaccharide pré-assemblé dans la membrane du réticulum endoplasmique ( $N$ glycosylation) et l'ajout de chaînes d'acides gras. En outre, au fur et à mesure de son allongement, la chaîne polypeptidique subit des repliements préludant à l'acquisition de sa structure tertiaire et empêchant l'agrégation entre elles des chaînes naissantes. Le repliement est assuré par des enzymes résidentes et contrôlé par des protéines également résidentes, les chaperons [10, 11]. Le réticulum endoplasmique est aussi le siège de mécanismes empêchant la progression vers le compartiment suivant de protéines incorrectement assemblées. Celles-ci sont soit retenues, soit dégradées sur place. Certaines d'entre elles peuvent même revenir dans le cytoplasme où elles sont dégradées par la voie ubiquitine-protéasome [16-18]. 
Ce très bref résumé des recherches qui se sont considérablement développées au cours du dernier quart du XXe siècle permet de dresser un « portrait » du réticulum endoplasmique. Il peut être comparé à une usine fabriquant des composants qui vont être transportés à des stations de sous-traitance spécialisées dans l'achèvement de produits « finis », produits qui seront ensuite dirigés vers leur destination finale.

\section{Le compartiment golgien modifie, trie et dirige les protéines transportées}

Les études morphologiques et fonctionnelles inaugurées au début des années 1960 ont rapidement montré que l'appareil de Golgi est une structure complexe aux frontières floues qui fut rapidement divisée en compartiments distincts, définis à la fois par leur position dans la cellule et leurs fonctions (Figures 2-4).

\section{Définition des sous-compartiments golgiens}

Classiquement, trois compartiments, cis, médian et trans sont identifiés (Figure 4), chacun correspondant à une étape de progression antérograde des protéines issues du réticulum endoplasmique. Schématiquement, ces compartiments sont représentés par trois citernes aplaties et parallèles entourées de vésicules (Figure 4). La réalité morphologique n'est pas aussi simple [19]. De nombreuses variations s'observent selon la spécialisation et l'état fonctionnel des cellules. Cependant, le caractère constant réside dans l'existence de quelques citernes aplaties et allongées, souvent recourbées et associées à une riche population de vésicules ou de tubules (Figures 3, 4).

À ces trois compartiments classiques s'ajoutent des régions frontières assurant respectivement la communication avec le compartiment précédent, le réticulum endoplasmique, et avec le compartiment suivant, le compartiment post-golgien. II s'agit, d'une part, du compartiment intermédiaire et, d'autre part, du réseau trans du Golgi (Figure 4). Le compartiment intermédiaire, en position cis, est morphologiquement très hétérogène selon les modèles cellulaires et les conditions expérimentales utilisées par les chercheurs. Cela explique qu'il ait reçu des dénominations diverses dans la littérature anglosaxone tels que CGN (cis-Golgi network), ERGIC (endoplasmic reticulumGolgi intermediate compartment), VTC (vesiculotubular compartment) ou encore salvage compartment [11]. À l'opposé, le réseau trans du Golgi, ou trans-Golgi network (TGN), défini par Griffith et Simons en 1986 [20], est une entité à la fois morphologique et fonctionnelle. Il est en général inclus dans le compartiment post-golgien.
Le compartiment golgien contient l'équipement enzymatique responsable de modifications des protéines transportées.

Dans le compartiment golgien, les protéines sont modifiées par l'addition covalente aux chaînes polypeptidiques de différents groupements tels que des chaînes glycosylées, des acides gras ou des groupements sulfates, et par des coupures protéolytiques. Ces modifications se produisent séquentiellement, au fur et à mesure de la progression des protéines. De même, les lipides membranaires peuvent être glycosylés, donnant naissance aux sphingolipides qui participent à la composition de la membrane plasmique (Figure 5). Les enzymes responsables de ces modifications sont membranaires ou luminales $[10,11]$. Nombre d'entre elles ont été clonées et localisées par des anticorps spécifiques.

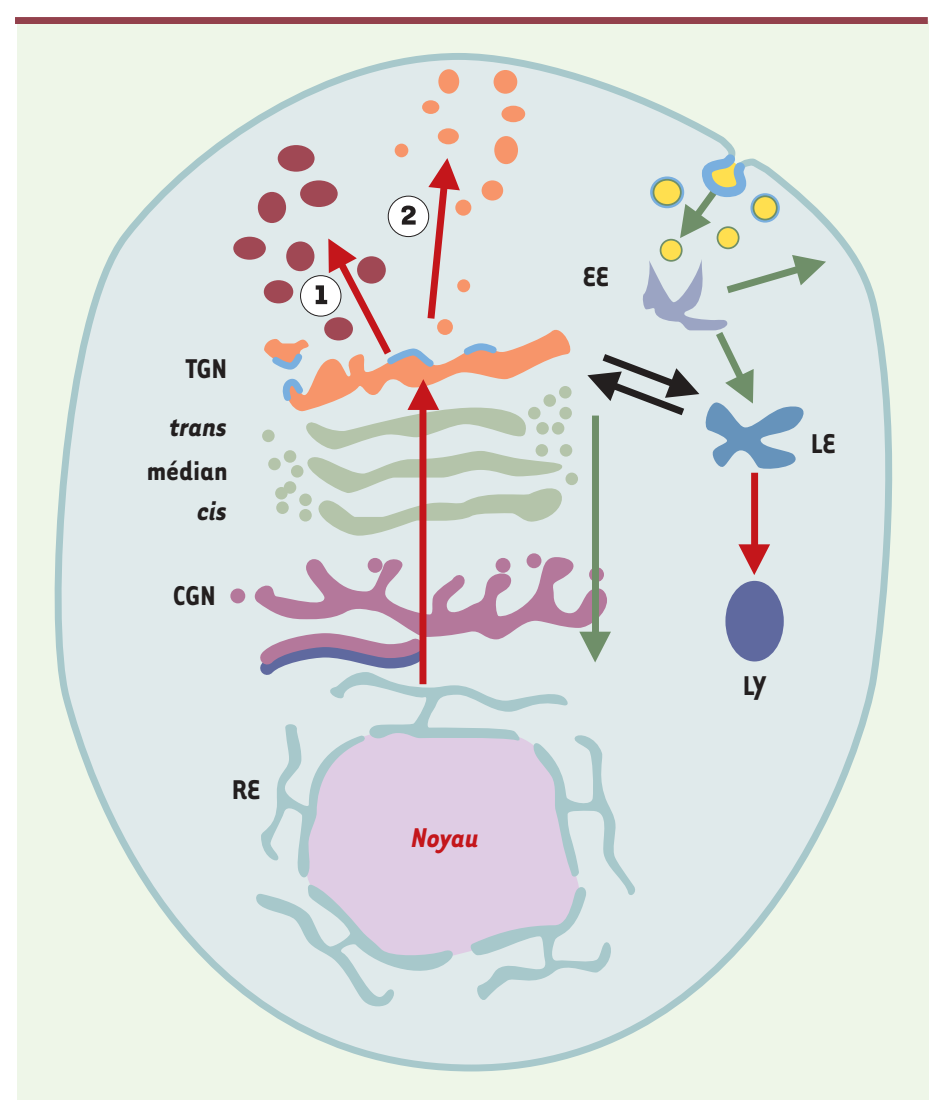

Figure 4. Schéma des compartiments cellulaires impliqués dans les voies de biosynthèse-exocytose et d'endocytose. La voie de biosynthèse est en rouge, celles d'endocytose est en vert. RE : réticulum endoplasmique ; CGN : cis-Golgi network ou compartiment intermédiaire ; TGN : trans-Golgi network. Les voies 1 et 2 sont respectivement les voies soumise à régulation et constitutive. La voie d'endocytose est en noir. દદ : early endosome ; LE : late endosome ; LY : lysosome. Le revêtement de clathrine est représenté en bleu clair sur le TGN et quelques vésicules d'endocytose. 
L’appareil de Golgi contient des protéines résidentes Outre des enzymes impliquées dans certaines des réactions décrites ci-dessus, d'autres protéines résidentes spécifiques des membranes de sous-compartiments golgiens ont été isolées. Plusieurs protéines sont spécifiques du compartiment cis ( $p 23 / 24$, p28, ...). La protéine TGN38 est spécifique du TGN. Ces protéines participent au contrôle du trafic intracellulaire.

L'existence de protéines membranaires spécifiques de sous-compartiments golgiens pose le problème des mécanismes assurant la permanence relative de leurs localisations respectives en dépit du flux membranaire nécessaire au transport antérograde des protéines exportées. Deux hypothèses principales ont été envisagées pour lesquelles des arguments expérimentaux convaincants ont été obtenus : le retour, en direction rétrograde, des protéines à leur site d'insertion par un mécanisme tubulo-vésiculaire, et l'existence de mécanismes intrinsèques liés à la structure de la protéine comme par exemple l'interaction du domaine transmembranaire avec des microdomaines lipidiques de la membrane, ou l'interaction du domaine cytoplasmique avec des protéines de l'environnement local (matrice) de l'appareil de Golgi [10, $11,21]$.

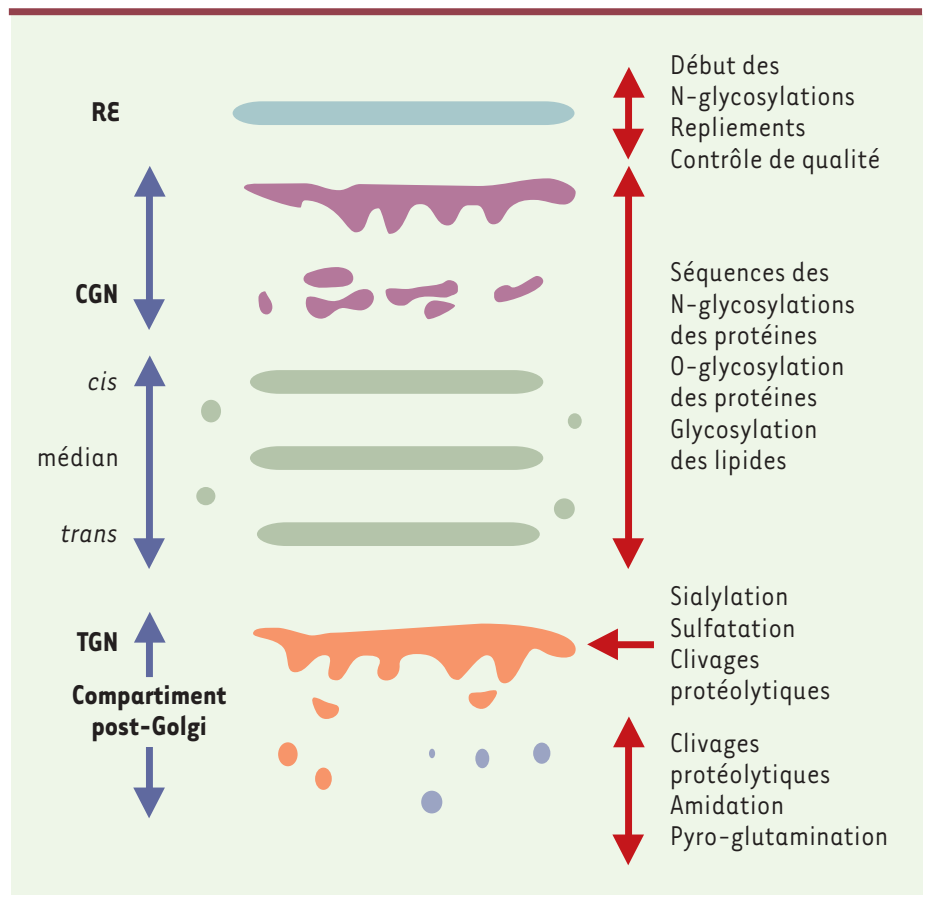

Figure 5. Principales modifications biochimiques des protéines au cours de leur transport antérograde dans les compartiments successifs. $R E$ : réticulum endoplasmique ; CGN : cis-Golgi network ou compartiment intermédiaire ; TGN : trans-Golgi network.
L'appareil de Golgi est un centre de tri et de ciblage Au cours de leur voyage dans les compartiments golgiens, les protéines sont triées. D'une part, elles ne subissent pas toutes les mêmes modifications, en fonction de leur structure et de leur interaction avec les éléments de l'environnement luminal. D'autre part, elles ne suivent pas toutes le même chemin. Certaines d'entre elles retournent en arrière, d'autres n'effectuent pas le parcours complet. Elles sont donc à la fois triées et dirigées. Les étapes ultimes du tri et du ciblage se déroulent dans le TGN où se situent en outre les dernières modifications enzymatiques.

\section{Le compartiment post-golgien est un carrefour}

Le compartiment post-golgien est exclusivement défini par sa localisation entre la citerne de l'appareil de Golgi et la membrane plasmique. En effet, contrairement aux compartiments précédents, il présente une grande diversité d'organisation en fonction du type cellulaire.

En outre, il est le lieu de rencontre de deux voies opposées du trafic membranaire : la voie centrifuge de biosynthèse-exocytose et la voie centripète d'endocytose. Le cytoplasme post-golgien contient donc les organites caractéristiques de ces deux voies qui se rejoignent à un même carrefour, le TGN (Figure 4). Le TGN se caractérise morphologiquement comme un réseau tubulaire polymorphe formant de nombreux bourgeons à la face trans du Golgi (Figure 3). Il a été identifié à l'origine par sa richesse en phosphatases acides et par la présence, à la surface cytoplasmique, de certains bourgeons d'un revêtement dense aux électrons identifié ensuite biochimiquement comme étant une protéine, la clathrine. En 1986, Griffith et Simons [20] ont proposé son rôle dans le tri et l'adressage des protéines issues de l'appareil de Golgi.

Les organites de la voie de biosynthèse-exocytose À la sortie du TGN, la voie de biosynthèse diverge en deux courants distincts : la voie soumise à régulation et la voie constitutive (Figure 4), définies après Tartakoff par Kelly [22]. La première voie est représentée par des organites stockés temporairement dans le compartiment post-golgien et dont l'exocytose est déclenchée par des signaux extracellulaires reconnus par des récepteurs spécifiques de la membrane plasmique. C'est donc une définition fonctionnelle. Les organites les mieux connus de cette voie sont les grains de sécrétion présents dans les cellules glandulaires et dans les neurones sécrétant des neuropeptides. La voie constitutive est définie par l'exportation directe de structures vésiculaires dont le trajet ne comporte aucun temps de rétention dans le cytoplasme. C'est une voie définie par défaut et qui correspond à un ensemble de structures de 
transport pléiomorphes. Les deux voies coexistent dans les cellules sécrétrices, et il a été clairement montré que, dans ce cas, elles sont séparées au niveau du TGN [23]. Une population vésiculaire spécifique des neurones est également issue du TGN : ce sont les vésicules synaptiques, qui sont transportées du TGN vers les synapses où elles acquièrent leur configuration et sont stockées avant de subir une exocytose réglée. Elles n'appartiennent donc pas à la voie constitutive.

L'identification biochimique des composants des organites de la voie de biosynthèse représente une étape indispensable pour l'étude de leur biogenèse et de leur tri. Du fait de la spécialisation des cellules glandulaires, les grains de sécrétion constituent une population très hétérogène et les mécanismes de leur biogenèse posent encore des problèmes [24]. Les vésicules synaptiques constituent en revanche une population morphologiquement et biochimiquement beaucoup plus homogène et offrent donc un modèle de recherche favorable. Leur membrane contient plusieurs protéines intégrales majoritaires (synaptophysine, synaptobrévine ou VAMP, synaptotagmine) ainsi que des protéines fonctionnelles assurant le maintien et la spécificité de composition de leur contenu. Les vésicules synaptiques ont servi de modèle à l'étude des mécanismes moléculaires de la fusion membranaire et de l'exocytose [25].

\section{Les compartiments de la voie d'endocytose}

La voie d'endocytose assure l'entrée dans la cellule de composants membranaires ou solubles destinés soit à leur dégradation dans les lysosomes, soit à leur adressage dans différents territoires de la cellule. La voie d'endocytose classique (Figure 4) comprend plusieurs compartiments que nous citons dans le sens centripète de leur fonctionnement : les puits d'endocytose tapissés de clathrine, les vésicules issues de la fission de ces puits, les endosomes précoces issus de la fusion des vésicules en un réseau dynamique de tubules et de vésicules, et les endosomes tardifs issus des précédents. Les endosomes précoces assurent en outre la dissociation des récepteurs internalisés et permettent leur recyclage à la membrane plasmique. Les endosomes tardifs communiquent à la fois avec le TGN et avec les lysosomes qui contiennent des hydrolases acides associées à des produits de dégradation des ligands dégradés et représentent le compartiment ultime de la voie d'endocytose classique [26]. D'autres voies d'entrée dans la cellule ont été plus récemment découvertes [27].

Comment concilier la spécificité de l'organisation et des fonctions des différents compartiments membranaires de la cellule eucaryote avec les mouvements centripète et centrifuge qui les animent constamment, c'est-à- dire avec le dynamisme inhérent à la vie de la cellule ? Cette question est au centre des recherches de biologie cellulaire depuis les 20 dernières années.

\section{Les implications en pathologie}

La recherche et l'identification de gènes responsables de maladies humaines a mis en évidence, dans de très nombreux cas, des mutations associées à une altération dans le transport intracellulaire de protéines d'importance physiologique. Près d'une centaine de maladies ont déjà été répertoriées et la liste n'est pas close [28]. Des défauts situés au niveau du réticulum endoplasmique ont été identifiés : la protéine mutée est soit dégradée sur place, soit stockée et incapable de poursuivre sa route. Ces erreurs d'adressage concernent des protéines membranaires comme le canal chlore dans la mucoviscidose, le récepteur des LDL dans l'hypercholestérolémie familiale, le récepteur de l'hormone de croissance dans le nanisme de Laron. Ils concernent aussi des protéines sécrétoires comme la thyroglobuline dans l'hypothyroïdie congénitale ou le précurseur de la vasopressine dans le diabète insipide. De nombreuses maladies sont liées à des défauts de la biogenèse ou du stockage des lysosomes. Plusieurs maladies auto-immunes sont dues à des autoanticorps dirigés contre des protéines ou des lipides impliqués dans le trafic vésiculaire [28]. Dans la plupart des cas, le lien entre ces mutations de gènes d'intérêt physiologique et leur ciblage à des partenaires connus du transport intracellulaire n'est pas établi. II est donc clair qu'une meilleure compréhension des mécanismes cellulaires de ces défauts est indispensable pour envisager de possibles interventions thérapeutiques.

En conclusion, le développement des recherches sur la dynamique des compartiments membranaires pour la compréhension du fonctionnement normal et pathologique des cellules apparaît donc au moins aussi capital que celui des recherches sur le génome humain. $\diamond$

\section{SUMMARY}

Membrane compartments in eukaryotic cells

The existence of an intracellular reticulum in eukaryotic cells was revealed at the middle of the XXth century by electron microscopic observations performed on living fibroblasts and then on cell thin sections. Two compartments were morphologically distinguished in this reticulum: the rough endoplasmic reticulum and the smooth endoplasmic reticulum which was identified to the "apparatus" previously described, by the end of the XIXth century, at the light microscope by Golgi. An important progress was then accomplished due to functional studies that demonstrated that these compartments are in continuous 
communication and that they are the site of the intracellular transport of exported proteins. This led to the concept of membrane traffic and to the hypothesis put forward by Palade of the existence of intracellular transport vesicles, budding and pinching off from donor membranes and fusing with acceptor membranes. Biochemical, molecular and genetic studies performed during the last quarter of the XXth century aimed at elucidating the structural features and functions of the various intracellular compartments. At the same time they have revealed the unicity of organization of all eukaryotic cells. The present chapter surveys the main properties of the intracellular membrane compartments and subcompartments involved in the exportation of proteins (the biosynthetic pathway) or in their importation (endocytic pathway). This knowledge represents necessary basis to the understanding of the most recent studies on the molecular mechanisms of intracellular transport. $\diamond$

\section{RÉFÉRENCES}

1. Garnier C. Les filaments basaux des cellules glandulaires. Bibliographie Anatomique 1897 ; V : 278-89.

2. Golgi C. Sur la structure des cellules nerveuses. Archives Italiennes de Biologie 1898 ; XXX: 61-71.

3. Dalton AJ, Felix MD. Cytologic and cytochemical characteristics of the Golgi substance of epithelial cells of the epididymus in situ, in homogenates and after isolation. Am J Anat 1954 ; 94 : 171-87.

4. Palade GE. Intracellular aspects of the process of protein secretion. Science 1975 ; 189 : 347-58.

5. Haguenau F. The ergastoplasm: its history, ultrastructure and biochemistry. Int Rev Cyt 1958 ; VII : 425-82.

6. Lippincott-Schwartz J, Snapp $\varepsilon$, Kenworthy A. Studying protein dynamic in living cells. Nat Rev Mol Cell Biol 2001 ; 2 : 444-56.
7. Mironov A, Weidman P, Luini A. Variations on intracellular transport theme: maturing cisternae and trafficking tubules. J Cell Biol 1997 ; 138 : 481-4.

8. Mironov A, Beznoussenko G, Nicoziani P, et al. Small cargo proteins and large aggregates can traverse the Golgi by a common mechanism without leaving the lumen of cisternae. J Cell Biol 2001 ; 155 : 1225-38.

9. Zerial M, Mc Bride H. Rab proteins as membrane organizers. Nat Rev Mol Cell Biol 2001 ; 2 : 107-17.

10. Alberts A, Bray D, Lewis D, et al. Biologie moléculaire de la cellule, $2^{\mathrm{e}}$ ed. Paris : Flammarion, 1995 : 1220 p.

11. Tixier-Vidal A. Biologie cellulaire de la sécrétion des protéines. Paris : Polytechnica, 1997 : 150 p.

12. Simons K, Toomre D. Lipid rafts and signal transduction. Nat Rev Mol Cell Biol 2000 ; 1 : 31-9.

13. Sprong $H$, van der Sluijs $P$, van Meer G. How proteins move lipids and lipids move proteins. Nat Rev Mol Cell Biol 2001 ; 2 : 504-13.

14. Singer $S$, Nicolson $G$. The fluid mosaic model of the structure of cell membranes. Science 1972 ; $175: 720-31$.

15. Blobel G. Protein targeting. Biosci Rep 2000 ; 20 : 303-44.

16. Ellgaard L, Helenius A. ER quality control: towards an understanding at the molecular level. Curr Opin Cell Biol 2001 ; 13 : 431-7.

17. Vashist S, Kein W, Balden W, et al. Distinct retrieval and retention mechanisms are required for the quality control of endoplasmic reticulum protein folding. J Cell Biol 2001 ; 155 : 355-68.

18. Weissman A. Themes and variations on ubiquitylation. Nat Rev Mol Cell Biol 2001 ; 2 : 169-78.

19. Rambourg A, Clermont $y$. Three-dimensional structure of the Golgi apparatus in mammalian cells. In : Berger \&G, Roth J, eds. The Golgi apparatus. Basel : Birkhauser Verlag, 1997 : 37-61.

20. Griffiths G, Simons K. The trans Golgi network: sorting at the exit site of the Golgi complex. Science 1986 ; 234 : 438-43.

20. Munro S. Localization of proteins to the Golgi apparatus. Trends Cell Biol 1998 ; 8 : 11-5.

22. Kelly R. Pathways of protein secretion in eukaryotes. Science 1986 ; 230 : 25-31.
23. Tooze S, Huttner W. Cell free protein sorting to the regulated and constituve secretory pathways. Cell $1990 ; 60: 337-47$.

24. Tooze S, Martens G, Huttner WB. Secretory granule biogenesis: rafting to the SNARE. Trends Cell Biol 2001; 11 : 116-22.

25. Hannah MJ, Schmidt AA, Huttner WB. Synaptic vesicle biogenesis. Annu Rev Cell Dev Biol 1999 ; 15 : 733-98.

26. Gruenberg J. The endocytic pathway: a mosaic of domains. Nat Rev Mol Cell Biol 2001 ; 2 : 721-30.

27. Gisou van der Goot F, Gruenberg J. Oiling the wheels of the endocytic pathway. Trends Cell Biol 2002 ; 12 : 296-8.

28. Aridor M, Hannan L. Traffic jam: a compendium of human diseases that affect intracellular transport process. Traffic 2000 ; 1 : 836-51.

29. Cajal SR. Algunas variaciones fisiológicas y patológicas del aparato reticular de Golgi. Trab Lab Invest Biol Univ Madrid 1914 ; 12 : 127-227.

30. Tougard C, Tixier-Vidal A. Lactotropes and gonadotropes. In : Knobil $\varepsilon$, Neill JD, eds. The physiology of reproduction. New York: Raven Press, 1994 : 1712.

\section{TIRÉS À PART}

A. Tixier-Vidal 